\title{
A pilot evaluation of an online cognitive behavioral therapy for insomnia disorder - targeted screening and interactive Web design lead to improved sleep in a community population
}

\author{
This article was published in the following Dove Press journal: \\ Nature and Science of Sleep \\ 17 March 2014 \\ Number of times this article has been viewed
}

\section{Kirstie N Anderson \\ Paul Goldsmith \\ Alison Gardiner}

Regional Sleep Service, Freeman Hospital, Newcastle upon Tyne, UK
Correspondence: Kirstie N Anderson Regional Sleep Service, Freeman Hospital, Newcastle upon Tyne NEI 4LP, UK

Tel +44 I 912823833

Fax +44 191 2333435

Email kirstie.anderson@nuth.nhs.uk
Introduction: Computerized or online cognitive behavioral therapies (CBTs) are increasingly being developed to deliver insomnia therapy (CBT-i). They seek to address the difficulty of delivering an evidence-based technology to a large number of patients at low cost. Previous online applications have shown significant but variable improvements in sleep efficiency and a decrease in insomnia severity when compared with control groups. The best online methodology remains debated, and there are no such applications currently available within the UK National Health Service.

Method: Evaluation of treatment outcomes in 75 participants with insomnia disorder using an open-access, novel, interactive online therapy. Rigorous screening was first undertaken to exclude those with probable sleep apnea, restless legs, circadian rhythm disorder, or significant anxiety or depression prior to commencing therapy. A modern interactive video-based website was used to encourage compliance by personalizing therapy based on response. Sleep efficiency, sleep latency, total sleep time, and sleep quality were all assessed prior to and after intervention.

Results: Of those who accessed therapy, $62 \%$ were excluded based on a likely diagnosis of another sleep disorder (788/1281). Participants who completed therapy all had severe insomnia disorder, with a group mean sleep efficiency of $55 \%$. After intervention there was a significant increase in sleep efficiency and sleep latency, with modest nonsignificant improvements in total sleep time. The majority of users reported improved sleep quality, and compliance with therapy was very good, with over $64 / 75$ completing $>90 \%$ of sleep diary entries.

Conclusion: Online CBT-i can be designed to deliver personalized therapy with good reported outcomes and high compliance rates in those who start therapy. This initial evaluation also suggests that screening for other sleep disorders and mental health problems is necessary as many other sleep disorders are detected in those who self-refer with insomnia. This would inform the development of any larger-scale applications within the psychological therapies used in the health care system.

Keywords: CBT, online applications, sleep efficiency, sleep latency, total sleep time, sleep quality, compliance

\section{Introduction}

Insomnia disorder ${ }^{1}$ is highly prevalent in the general population, and a number of epidemiological studies estimate prevalence at $10 \%-12 \%{ }^{2,3}$ Defined as difficulty initiating sleep, difficulty maintaining sleep, with subsequent impact upon daytime function, insomnia disorder is associated with daytime fatigue, reduced quality of 
life, and increased ill health across a range of studies. ${ }^{4,5}$ Insomnia predicts subsequent depression or anxiety in those with a first episode of mood disorder ${ }^{6,7}$ and is also associated with physical health problems such as hypertension and diabetes. ${ }^{8,9}$ However, persistent insomnia often goes unrecognized in primary care, and there are few well-developed care pathways. ${ }^{10,11}$

Pharmacological therapies are widely used in primary care, but there is limited evidence for their benefit in chronic insomnia and increasing evidence that these medications are associated with long-term risks, particularly in an older population. ${ }^{12,13}$ There is a large body of evidence, based on over 20 years of randomized controlled trials (RCTs), for a specific form of cognitive behavioral therapy (CBT), CBT for insomnia (CBT-i), being an effective and long-lasting treatment for $50 \%-70 \%$ of patients. ${ }^{14,15}$

CBT-i tackles factors that maintain insomnia over time, including sleep-related dysfunctional cognitions, behaviors which perpetuate insomnia, and dysregulation of the homoeostatic sleep drive. Recording and interpreting sleep diaries is key. The efficacy of CBT-i to improve outcomes in primary and comorbid insomnia patients has been repeatedly demonstrated, with improvements in both mental and physical health outcomes. ${ }^{16-18}$

Despite widespread acceptance of CBT-i as an effective therapy, the current challenge remains that of scaling therapy to population need and developing cost-effective and accessible care pathways that could be implemented within health care systems. CBT as a face-to-face therapy remains a costly option. The current model of Immediate Access to Psychological Therapies (www.iapt.nhs.uk) within the UK allows increased access to psychological therapy but still remains dependent on regular contact with patients.

Online therapies are increasingly available to the general public for a variety of disorders and offer the possibility of personalized but cost-effective therapies. Several smallcohort studies and two RCTs to date have evaluated online CBT-i and shown promising results but with varying effect sizes. ${ }^{19-22}$ The most effective and responsive Web-based system remains debated.

Alternative, undiagnosed causes of sleep disturbance, such as depression, anxiety, sleep apnea, and restless legs, may account for lower success rates compared with more rigorous face-to-face screening. It is also important for any online therapy to respond to the increasing competition from a very wide variety of Internet resources for health and to make use of the most up-to-date Web design and interactive Web technology. Therapies must be able to monitor outcomes based on evidence-based parameters such as sleep efficiency (SE) and total sleep time (TST). Any online therapy must also be flexible and replicate the interaction with a face-to-face therapist as closely as possible.

We developed a novel, interactive video-based, online CBT-i program that required participants to complete screening for other sleep disorders prior to commencing therapy. The therapy was designed to respond to sleep diary entries and to alter advice based on responses but also to allow direct support from medically trained staff. We hypothesized that this would enrich those with insomnia disorder and therefore improve outcome measures. The program was deliberately made available to the general public without prior screening but was developed by a specialist sleep service (Regional Sleep Service, Freeman Hospital, UK) with much experience of CBT-i.

\section{Methods}

\section{Subjects}

Participants were all over 18 years of age, and there was no upper age limit. They were recruited from the UK general public over a 6-month period, and without prior screening, from a website advertising an evidence-based, medical therapy for insomnia supervised by an NHS sleep-medicine specialist.

All were $>18$ years and fulfilled the Diagnostic and Statistical Manual of Mental Disorders, Fifth Edition(DSM-5) criteria for persistent insomnia disorder lasting $>3$ months. All enrolling participants had to complete a set of online screening questions. The ongoing use of sleeping pills or other sleep aids was permitted. Usual care that participants had been receiving via their medical advisers, including medical prescriptions, continued. The website http://www. sleepstation.org.uk hosts examples of the illustrative material of the study and describes the intervention procedures.

\section{Screening}

Prior to commencement of therapy, all participants were required to complete an Epworth Sleepiness Score, ${ }^{23}$ a validated score of sleepiness in association with sleep apnea and the Pittsburgh Sleep Quality Index (PSQI), ${ }^{24}$ a widely used scale of subjective sleep disturbance with a PSQI $>5$ defined as abnormal. They also completed the 21-item assessment of the Depression Anxiety Stress Scale (DASS-21) ${ }^{25}$ and were specifically questioned about snoring, witnessed apneas, and restless legs syndrome, with video explanations of these disorders. Specifically, subjects were asked about loud snoring on a nightly basis that disrupted 
partners and could be heard in another room, with witnessed prolonged pauses in breathing and daytime sleepiness. The definition of restless legs syndrome was as per the International Restless Legs Syndrome Society ${ }^{26}$ definition, with an unpleasant sensation in the legs, which is worse in the evening, causing the desire to move, and is relieved partially or completely by movement.

Exclusion criteria were those with: an Epworth Sleepiness Score $>11$; DASS-21 scores in the severe or extremely severe range for either depression, anxiety, or stress; and possible obstructive sleep apnea syndrome and/or possible moderate or severe restless legs syndrome. These patients were excluded from further participation and were asked to consult their regular medical practitioner to discuss appropriate medical therapy prior to enrolling in the program.

All patients then prospectively completed 2 weeks of online sleep diaries to help confirm the diagnosis of insomnia disorder. For this analysis, participants had to have a mean (baseline) SE (defined as TST per time in bed, in hours) $\leq 80 \%$ to reflect insomnia of sufficient clinical severity to assess for benefit on our primary outcome variable of improvement in SE.

A total of 1,281 people initially registered their interest in the program, and of these, 493 completed all the screening questionnaires and were considered eligible to complete 2 weeks of sleep diaries to further evaluate their sleep patterns. Of this group, 151 completed 2 weeks of sleep diaries to further look at their patterns, and 75 patients were formally evaluated having completed the 6-8 week therapy program and fulfilling the criteria for clinically significant insomnia disorder. Of those who were not evaluated, 25 had SE $>80 \%$, and insomnia was not considered of sufficient severity for this evaluation. These 25 were given the same information about sleep hygiene and regular schedules but also information regarding sleep misperception. They were allowed to complete the treatment program if they wished, and 17/25 with SE $>80 \%$ did so. However, their outcome data is not reported here.

Sixteen subjects had diaries suggestive of circadian rhythm disorder, including shift work, and were excluded; 34 did not commence therapy (six due to intercurrent illness and six due to spontaneous improvement in symptoms).

To ensure real-world evaluation of the online intervention, all participants were enrolled and treated via email contact and the website only. All eligibility and baseline sleep questionnaire data were also automatically obtained without face-toface verification. Ineligible participants were provided with access to a number of leaflets about common sleep disorders and tailored sleep hygiene advice and information. Any extra technical or therapy support required by participants was provided by an email support system. The website was commercial, but the group evaluated undertook the course of therapy at no cost to themselves, they did not receive any incentive to complete therapy.

\section{Details of therapy and assessment}

Following completion of 2 weeks of online sleep diaries, all patients accessed an online sleep diary throughout the remainder of the study, which they completed daily. They received an automated 6-8-week therapy program consistent with the published treatment manuals, ${ }^{27}$ comprising sleep restriction (automatically calculated based on SE but never $<5$ hours), cognitive control, stimulus control techniques, and sleep hygiene education. Patients completed sleep diaries online throughout and treatment was tailored to responses on a weekly basis. Therefore, although all patients received the same components of therapy, the treatment was personalized based on both their diary responses, speed of improvement, and pattern, eg, differing amounts of sleep restriction, advice regarding daytime napping, and clock watching. This allowed very flexible therapy to replicate face-to-face therapy as closely as possible. The system automatically detected whether diary entries were being made daily, prompted subjects if they missed an entry, and offered further support if necessary.

SE (as TST per time in bed, in hours) and TST were automatically calculated from sleep diaries. Patients had access to both video-delivered therapy advice and written PDF (portable document format) documents that they could print out. Patients who progressed slowly or had difficulty with any aspects of the program and felt they needed longer were allowed to continue with the therapy program for up to 10 weeks (and 15 in a single case) as long as SE was clearly still improving. However, for consistency and for the purposes of this paper, all data were analyzed after 8 weeks of therapy.

Patients completed items (eg, light-out time, time to fall asleep or sleep latency [SL], duration of night wakenings, and wake after sleep onset) by selecting times in 15-minute time increments from dropdown menus, so that SE could be calculated (TST per time in bed). Diary data were assessed at baseline, post-treatment, and 8 weeks after treatment. The primary endpoint was change in SE from pre-treatment to posttreatment. Changes in wake after sleep onset, SL, and TST were also calculated. SE was used to provide an overall measure of insomnia by measuring both difficulty falling and staying asleep and remains the most widely published primary outcome in other similar studies. ${ }^{28}$ Patients could view their SE and TST at the end of each week. Patients were also asked to report upon qualitative improvement of sleep by simply answering whether 
they felt sleep quality was unchanged, worse, improved, or greatly improved after completion of therapy. These outcome measures were made 4 weeks after completion of therapy. Figure 1 shows representative sleep diaries from a participant's sleep diary before, during, and upon completion of therapy.

\section{Statistical analysis}

The SE, TST, and SL scores were non-normally distributed (Kolmogorov-Smirnov test for normality), and therefore, significance was tested using the Wilcoxon signed-rank test.

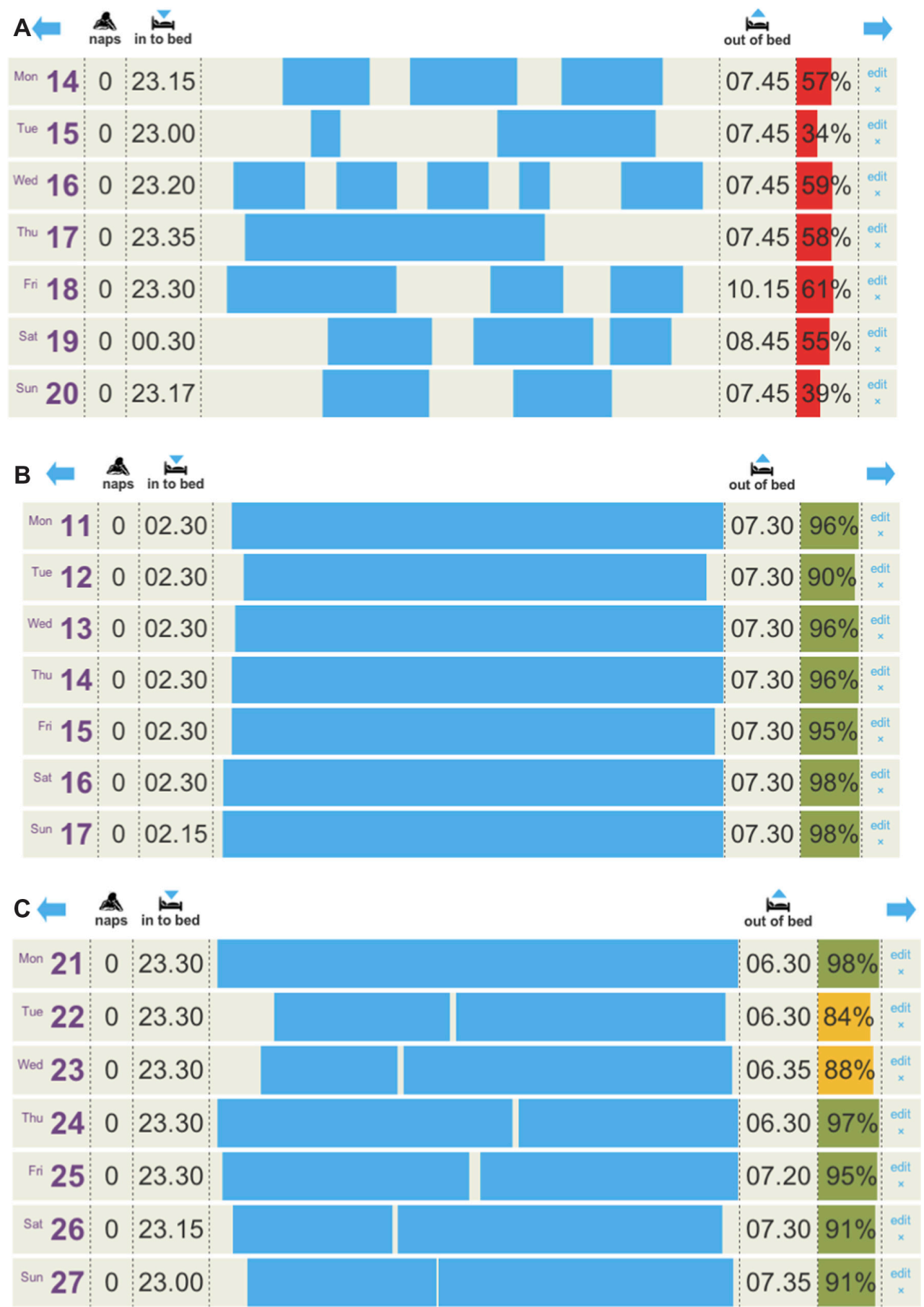

Figure I Three sleep diaries are shown, the data were entered by the participants, and the sleep efficiency was automatically calculated based on time into bed and reported time asleep. The day, date, and any daytime naps are also shown. Sleep efficiency below $80 \%$ is shown in red, below $90 \%$ is shown in yellow, and above $90 \%$ is shown in green.

Notes: (A) Shows the second week of initial sleep diaries; (B) shows the period of sleep restriction where the participant has been instructed to keep time into bed to between 02.30 and $07.30 ;(\mathbf{C})$ shows the diary upon completion of 6 weeks of therapy. 


\section{Results}

\section{Participants}

Of the 75 participants, there were 48 female and 27 male ( $67 \%$ female, $33 \%$ male). The age range was $18-70$ (mean age 49 years). Prescribed medication to aid sleep (temazepam, $n=4$; amitriptyline, $n=7$; and zopiclone, $n=1$ ) was used at the time of therapy in 12 patients; four described using other methods to improve sleep (eg, relaxation tapes). Patients were advised to avoid, if possible, alteration of prescriptions during therapy, and to the best of our knowledge, only one patient stated that they had stopped a hypnotic during the therapy period.

All participants had evidence of DSM-5 insomnia disorder based on questionnaire screening responses and initial sleep diary data; the vast majority had both difficulty initiating and maintaining sleep. Fifteen subjects described having insomnia for more than 10 years, and seven for more than 20 years. Compliance with diary data was good, with $65 / 75$ completing all or $>90 \%$ of diary entries throughout therapy and all patients completing $75 \%$ of daily diary entries. All patients completed 2 weeks of sleep diaries prior to enrolling within the program; there were no significant differences between self-reported mean SE in week 1 compared with the mean SE over 2 weeks (55\%).

\section{Response to therapy}

The average duration of therapy following the initial 2 weeks of sleep diaries was 6.8 weeks (range 4-15). The program allowed those making slower progress the option of continuing to complete sleep diaries while SE was improving or where some aspects of the program were not being correctly followed, eg, due to holidays or changes in schedules. Eight people did this.

The median self-reported SE at entry was 55\% (range 14-79), reflecting insomnia in the severe range. The median $\mathrm{SE}$ at completion of therapy was $81 \%$ (range $53-95$ ). SE was significantly higher post-therapy than pre-therapy, $z=-7.433$, $P<0.05, r=-0.60$.

Of the 75 participants, 59 (79\%) improved SE to $\geq 20 \%$ from baseline. As all participants evaluated required initial $\mathrm{SE}<80 \%$, it was important to determine the proportion who exceeded this value ( $\mathrm{SE} \geq 80 \%$ ) following intervention. Of those completing therapy, $72 \%(54 / 75)$ had SE $>80 \%, 38 \%$ (29/75) had SE $>85 \%$, and $27 \%(20 / 75)$ had SE $>90 \%$.

The median TST before treatment averaged 4.7 hours per night (range 1.9-6.8), and the average TST after treatment had increased slightly to 5.3 hours (range 2.8-7.6). Sleep time was not significantly higher post-therapy than pre-therapy, $z=-1.294, P<0.05, r=-0.10$. The median SL before treatment was 76 minutes (range 24-168), and after treatment it had decreased to 21 minutes (range 6-100). Sleep latency was significantly lower post-therapy, $P<0.05$.

Few patients recorded daytime naps; $67 / 75$ reported no daytime naps but those who did, did not alter over the course of treatment. All were given advice about avoidance of daytime naps where possible as part of the therapy.

Sleep-diary data are summarized in Table 1.

Patients were asked to complete a simple assessment and report whether they felt sleep was "worse, unchanged, improved or greatly improved after completion of therapy". A total of 46 patients reported that sleep was much improved, 19 reported some improvement, eight felt sleep was no better, and two reported worsening sleep.

The PSQI prior to commencing therapy was 11.5 (range 8-18), and the mean PSQI after completion of therapy was 7 (range 3-15).

\section{Discussion}

We evaluated a novel, open-access, online CBT for insomnia therapy and demonstrated large improvements in our primary endpoint of SE. There were also clear improvements in SL and more modest improvements in TST. However, screening removed many who initially enrolled for therapy with other sleep disorders (such as suspected sleep apnea or restless legs syndrome) or significant depression, stress, and anxiety symptoms. Once participants fulfilled criteria for insomnia and had commenced therapy, the attrition rate was low, and compliance with sleep diaries and therapy recommendations was high. Sleep quality improved based on PSQI scores and self-reports from patients. There was marked variability in speed of improvement and how the participants used the system, in particular, how much individual support was required.

This study is one of a number to demonstrate that using the Internet to deliver an online version of CBT-i can improve

Table I Outcome data based on sleep diary for 75 patients who completed online insomnia therapy

\begin{tabular}{lll}
\hline & Pre-treatment & Post-treatment \\
\hline Sleep efficiency & $55.1 \% \pm 16.9 \%$ & $81.0 \% \pm 12.9 \%$ \\
Sleep latency (minutes) & 76 (range 24-168) & 21 (range 6-100) \\
$\begin{array}{l}\text { Total sleep time (hours) } \\
\text { Participants with sleep }\end{array}$ & $4.7 \pm 1.9$ & $5.3 \pm 1.3$ \\
efficiency $>80 \%$ & $0 / 75$ & $46 / 75$ \\
$\begin{array}{l}\text { Participants with sleep } \\
\text { efficiency }>90 \%\end{array}$ & $0 / 75$ & $20 / 75$ \\
\hline
\end{tabular}


SE and contribute to overall improvement in sleep. ${ }^{19-22,29,30}$ Therefore, an Internet-based CBT-i program may be an effective and relatively cheap first step in providing insomnia treatment for adults. A recent systematic review of these therapies found and evaluated six published RCTs ${ }^{21}$ (two from the same group, with the same online program) and there has been one further UK RCT published since then. ${ }^{19}$

Generally, the numbers treated within the published literature have been small. For the SHUT-i program evaluated in those with primary and comorbid insomnia in the US, $, 20,30$ group sizes were 22 treated participants and 14 participants, respectively, compared with waiting list controls. They showed improvements in SE of $16 \%$ and $19 \%$, but participants volunteered for the studies, were telephone-screened first, and were paid to complete the study. For the comprehensive study published by Espie et al in 2012 using an animated personal therapist, ${ }^{19} 164$ adults were randomly assigned to online CBT (55), image rehearsal therapy (55), and treatment as usual (54). It should be noted that this group came from a much larger group of 1,342 who fitted potential criteria for recruitment but 276 who responded to direct contact and 164 who were excluded following screening for further disorders. This again highlights the need to incorporate screening for other sleep disorders within any online therapy. A total of 43 out of 55 completed the full course of therapy, and of those, 40 completed an 8-week follow-up assessment, showing a $19.5 \%$ improvement in SE.

Given the established benefit of face to face intervention, this suggests that the variable treatment effect sizes may depend on the particular website design and delivery.

Our therapy had several novel features compared with previously described interventions. We used a real-world, open-access program with the novel features of inbuilt screening, the possibility of a support system, and the entirely novel feature of a system that was able to respond to the speed at which users went through the program to deliver information in a different fashion for different users. We used the instructional design models already published to utilize the flexibility of the Internet. ${ }^{31}$ This therapy also utilized features reported as successful in other programs, including engaging graphics, sleep diaries with daily data visualization reported to be easy to complete, and interactive videos throughout the process featuring a real therapist (KNA). An email support system allowed an individual response to be given if patients were having difficulties, and this proved very popular with participants; some reported that support enabled them to continue difficult aspects of therapy such as sleep restriction. However, this feature does potentially reduce the ability to more widely disseminate the program without an increase in cost.

In fact, the percentage of improvement in SE in our study is the highest published for this type of intervention. This is also the largest UK group studied with an online intervention accessible to all without prior screening. Clearly, further studies are required to directly compare face-to-face therapy with online therapy. We did not have a control group of those who complained of insomnia but remained untreated, and this remains a limitation, but this pilot data is encouraging. We also accepted the information provided by patients without access to general practitioner records and, again, accept this limitation within the data; however, the relative lack of insomnia service provision within the UK may well require a scalable treatment modality that screens at the point of entry into therapy and does not rely upon variable knowledge of sleep disorders within primary and secondary care.

Further potential limitations of this study include the lack of long-term outcome data, and also the fact that a number of patients were using other sleep aids. However, patients were advised to avoid alteration of prescriptions during therapy, and we felt this reflected real-life insomnia treatment, where many people try a number of possible therapies for insomnia over time. Ideally, these limitations would need to be addressed in a larger study with a control group. This study had a relatively low attrition once patients had commenced therapy. Only three patients (7\%) who reported persistent insomnia disorder discontinued therapy once it had started, and this is comparable to other studies. It is lower than some other reported groups, and targeted screening may have helped to ensure that those who started were those best suited to the therapy. The motivation of those who respond to online recruitment may not be representative of the larger population of those with insomnia, and further studies using Sleepstation are needed to evaluate outcomes within primary or secondary care settings. It is of note that a large proportion of those fulfilling criteria for insomnia disorder based on screening and diaries did not commence therapy, and this is also reflected in other studies where the proportion of people who completed therapy was far smaller than those initially screened and found to be suitable for recruitment. ${ }^{19}$

However, these outcomes are comparable in magnitude to therapist-delivered CBT and greater than the majority of online CBT studies within formal trials of interested subjects. Therefore, increasing sophistication and instructional design will be important for future online CBT applications. They may well represent an easily accessible and low cost form of therapy for this common but undertreated condition. 
However, careful participant screening and clear outcome measures will be necessary for health professionals to use these therapies, and further work is needed to improve compliance and treatment outcomes.

\section{Disclosure}

This was not an industry-supported study. The software and Web development for this study was supported by Rubrum Limited (Newcastle upon Tyne, UK). KN Anderson, P Goldsmith, and A Gardiner have not received any income from the company but are shareholders. There are no other financial conflicts of interest.

\section{References}

1. American Psychiatry Association. Diagnostic and Statistical Manual of Mental Disorders. 5th ed. Arlington, VA: American Psychiatric Publishing; 2013.

2. Ohayon MM. Epidemiology of insomnia: what we know and what we still need to learn. Sleep Med Rev. 2002;6(2):97-111.

3. Wilsmore BR, Grunstein RR, Fransen M, et al. Sleep habits, insomnia, and daytime sleepiness in a large and healthy community-based sample of New Zealanders. J Clin Sleep Med. 2013;9(6):559-566.

4. Espie CA, Kyle SD, Hames P, Cyhlarova E, Benzeval M. The daytime impact of DSM-5 insomnia disorder: comparative analysis of insomnia subtypes from the Great British Sleep Survey. J Clin Psychiatry. 2012;73(12):1478-1484.

5. Roth T, Ancoli-Israel S. Daytime consequences and correlates of insomnia in the United States: results of the 1991 National Sleep Foundation Survey. II. Sleep. 1991;22 Supp1 2:S354-S358.

6. Cole MG, Dendkuir N. Risk factors for depression amongst elderly community subjects: a systematic review and meta-analysis. $\mathrm{Am} \mathrm{J}$ Psych. 2003;160(60):1147-1156.

7. Baglioni $\mathrm{C}$, Battagliese $\mathrm{G}$, Feige $\mathrm{B}$, et al. Insomnia as a predictor of depression: a meta-analytic evaluation of longitudinal epidemiological studies. J Affect Disord. 2011;135(1-3):10-19.

8. Vgontzas AN, Liao D, Bixler EO, Chrousos GP, Vela-Bueno A. Insomnia with objective short sleep latency is associated with a high risk for hypertension. Sleep. 2009;32(4):491-497.

9. Vgontzas AN, Liao D, Pejovic S, Calhoun S, Karataraki M, Bixler EO. Insomnia with objective short sleep duration is associated with type 2 diabetes: a population based study. Diabetes Care. 2009;32(11): 1980-1985.

10. Cheung JM, Bartlett DJ, Armour CL, Glozier N, Saini B. Insomnia patients' help-seeking experiences. Behav Sleep Med. 2013;11:20

11. Sateia MJ, Nowell PD. Insomnia. Lancet. 2004;364(9449):1959-1973.

12. Curran HV, Collins R, Fletcher S, Kee SC, Woods B, Lliffe S. Older adults and withdrawal from benzodiazepine hypnotics in general practice: effects on cognitive function and quality of life. Psychol Med. 2003;33(7):1223-1237.

13. Bain KT. Management of chronic insomnia in elderly persons. Am J Geriatr Pharmacother. 2006;4(2):168-192.
14. Morin CM, Bootzin RR, Buysse DJ, Edinger JD, Espie CA, Lichstein KL. Psychological and behavioral treatment of insomnia: update of the recent evidence (1998-2004). Sleep. 2006;29(11):1398-1414.

15. Mitchell MD, Gehrman P, Perlis M, Umscheid CA. Comparative effectiveness of cognitive behavioral therapy for insomnia: a systematic review. BMC Fam Pract. 2012;25(13):40.

16. Belleville G, Cousineau H, Levrier K, St-Pierre-Delorme MÈ. Meta-analytic review of the impact of cognitive behavioral therapy for insomnia on concomitant anxiety. Clin Psychol Rev. 2011;31(4):638-652.

17. Kapella MC, Herdegen JJ, Perlis ML, et al. Cognitive behavioral therapy for insomnia comorbid with COPD is feasible with preliminary evidence of positive sleep and fatigue effects. Int J Chron Obstruct Pulmon Dis. 2011;6:625-635.

18. Pigeon WR, Moynihan J, Matteson-Rusby S, et al. Comparative effectiveness of CBT interventions for co-morbid chronic pain \& insomnia: a pilot study. Behav Res Ther. 2012;50(11):685-689.

19. Espie CA, Kyle SD, Williams C, et al. A randomized, placebo-controlled trial of online cognitive behavioral therapy for chronic insomnia disorder delivered via an automated media-rich web application. Sleep . 2012;35(6):769-781.

20. Ritterband LM, Thorndike FP, Gonder-Frederick LA, et al. Efficacy of an Internet-based behavioral intervention for adults with insomnia. Arch Gen Psychiatry. 2009;66(7):692-698.

21. Cheng SK, Dizon J. Computerised cognitive behavioural therapy for insomnia: a systematic review and meta-analysis. Psychother Psychosom. 2012;81(4):206-216.

22. Ström L, Pettersson R, Andersson G. Internet-based treatment for insomnia: a controlled evaluation. J Consult Clin Psychol. 2004;72(1): 113-120.

23. Johns MW. A new method for measuring daytime sleepiness: the Epworth sleepiness scale. Sleep. 1991;14(6):540-545.

24. Buysse DJ, Reynolds CF 3rd, Monk TH, et al. Quantification of subjective sleep quality in healthy elderly men and women using the Pittsburgh Sleep Quality Index (PSQI). Sleep. 1991;14(4):331-338.

25. Lovibond PF, Lovibond SH. The structure of negative emotional states: comparison of the Depression Anxiety Stress Scales (DASS) with the Beck Depression and Anxiety Inventories. Behav Res Ther. 1995;33(3): 335-343.

26. Allen RP, Picchietti D, Hening WA, Trenkwalder C, Walters AS, Montplaisi J. Restless legs syndrome diagnosis and epidemiology. A report from the restless legs syndrome diagnosis and epidemiology workshop at the National Institutes of Health. Sleep Med. 2003;4(2):101-119.

27. Perlis ML, Jungquist C, Smith MT, Posner D. Insomnia: A Session by Session Guide. 2nd ed. New York: Springer; 2008.

28. Morin CM. Measuring outcomes in randomized clinical trials of insomnia treatments. Sleep Med Rev. 2003;7(3):263-279.

29. Lancee J, van den Bout J, van Straten A, Spoormaker VI. Internetdelivered or mailed self-help treatment for insomnia?: a randomized waiting-list controlled trial. Behav Res Ther. 2012;50(1):22-29.

30. Ritterband LM, Bailey ET, Thorndike FP, et al. Initial evaluation of an Internet intervention to improve the sleep of cancer survivors with insomnia. Psychooncology. 2012;21(7):695-705.

31. Hilgart MM, Ritterband LM, Thorndike FP, Kinzie MB. Using instructional design process to improve design and development of Internet interventions. $J$ Med Internet Res. 2012;28(14)3:e89.
Nature and Science of Sleep

\section{Publish your work in this journal}

Nature and Science of Sleep is an international, peer-reviewed, open access journal covering all aspects of sleep science and sleep medicine, including the neurophysiology and functions of sleep, the genetics of sleep, sleep and society, biological rhythms, dreaming, sleep disorders and therapy, and strategies to optimize healthy sleep. The journal welcomes

\section{Dovepress}

original research, clinical \& epidemiological studies, reviews \& evaluations, case reports and extended reports. The manuscript management system is completely online and includes a very quick and fair peerreview system, which is all easy to use. Visit http://www.dovepress.com/ testimonials.php to read real quotes from published authors. 